Proceedings of the 2005 Winter Simulation Conference

M. E. Kuhl, N. M. Steiger, F. B. Armstrong, and J. A. Joines, eds.

\title{
SIMULATION BASED EVALUATION OF INFORMATION-CENTRIC SUPPLY CHAINS
}

\author{
Srinagesh Gavirneni \\ Johnson Graduate School of Management \\ Cornell University \\ Ithaca, NY 14853, U.S.A.
}

\begin{abstract}
In this paper we evaluate, via the use of simulation, information-centric design of three supply chains that are ubiquitous in operations management literature. With emphasis on inventories, we show how such a redesign of the supply chains can significantly enhance the benefit from information flows that are commonplace in the current business world. The underlying analysis is in the realm of stochastic non-stationary capacitated inventory control and the mathematical intractability behooves us to make extensive use of simulation. We postulate that the lack of such information centric re-designing of supply chains is one of the main reasons behind the failure, reported in popular press, of most information gathering projects in the industry.
\end{abstract}

\section{INTRODUCTION}

Recent enhancements in information technology have played a major role in the timely availability and accuracy of information across the supply chain. It is now cheaper to gather, store, and analyze vast amounts of data and this has presented managers with new opportunities for improving the efficiency of their supply chains. In addition, the latest developments in supply chain management have led everyone to believe that cooperation between members of a supply chain can lead to larger profits. While some gains have been realized from these developments, most organizations have failed to take the most advantage of them.

There have been reports, from industrial sources, of differing reactions to Electronic Data Interchange (EDI) benefits - while some were very happy with improved information, others were disappointed at the benefits (see Armistead and Mapes 1993 and Takac 1992). The popular press is full of stories about companies disillusioned with their Enterprise Resource Planning (ERP) systems. It is estimated that $70 \%$ of all ERP implementations do not recoup their investments and are branded as failures (see Lewis 2001). While there could be many reasons for this high failure rate, the fact that companies are not adept at using the information provided by these ERP systems is a major factor. Since the availability and accuracy of information are the key contributions of such enterprise-wide systems, the organizations must position themselves to benefit from it. It appears that, in order to utilize the information fully, there is a need for a firm to redesign its supply chain with regards to its structure and modus operandi.

The current research in supply chain management has failed to adequately answer the following important question: How should the supply chain structure and operating policies be changed in order to obtain the maximum benefit from these information flows? Many studies incorporated information into the existing setup and none considered changing the structure and/or the operating procedures in order to make better use of the information. We believe that such a change must be considered if one wants to take full advantage of the information. There is a need for analysis of these supply chains centered on the inherent information flows. Such an information-centric design and management of capacitated supply chains will address the issues of (i) How does one incorporate information flows into the decision making process? (ii) How does one determine which information is useful and worth gathering? How much money can be invested in collecting the information? and (iii) How should the supply chain structure and operating policies be changed in order to make the best use of the information flows?

We will demonstrate the benefits of information centric design and management of supply chains using three different supply chain configurations. These examples were chosen to capture the presence of (i) significant setup or ordering costs; (ii) price fluctuations; and (iii) inventory allocation issues. These three characteristics of supply chains were identified by Lee, Padmanabhan, and Whang (1996) as the main reasons for information distortion and supply chain inefficiency. In section 2 , we study a two stage supply chain with one supplier and one retailer facing end-customer demands. There is a significant ordering cost present at the retailer. Section 3 describes a two stage supply chain with 


\section{Gavirneni}

a single supplier and a single retailer (facing i.i.d. endcustomer demands) in which the supplier is charging the same price in every period. A single supplier, multi-retailer system is modeled and analyzed in section 4. For these three different supply chain configurations, we will propose, analyze, and compute the benefits of appropriate information centric policies that will significantly improve their performance. Section 5 contains ideas for future research and some closing remarks.

\section{TWO-STAGE SERIAL SUPPLY CHAIN WITH FIXED COSTS AT THE RETAILER}

Consider a supply chain containing one capacitated supplier and a retailer facing i.i.d. demands for a single product. The supplier has finite production capacity, $C$. The end-customer demand has cumulative distribution function (cdf) $\Psi(\cdot)$ and probability distribution function (pdf) $\psi(\cdot)$. The holding and penalty costs at the retailer are $h_{r}$ and $p_{r}$ respectively. They are $h_{s}$ and $p_{s}$ at the supplier. The costs and the demand distributions are known to both parties. There is a fixed ordering cost $K$ between the retailer and the supplier. There are no lead times either at the retailer or at the supplier. The unsatisfied demands at the retailer are backlogged and the unsatisfied demands at the supplier are sent to the retailer using an expediting (e.g. overtime) strategy and $p_{s}$ represents the cost of expediting. Thus, if needed, the retailer can order and receive an infinite quantity of the product in a period. All these assumptions are common in inventory control literature and in spite of its simple setup, this two stage supply chain can provide valuable insights into managing more complex systems efficiently. Cachon and Zipkin (1999), Gavirneni, Kapuscinski, and Tayur (1999), and Gavirneni and Tayur (1999) have used settings similar to this one to understand the effect of cooperation on inventories in supply chains.

The sequence of events in this supply chain is as follows. (1) The supplier decides on her inventory level restricted by her production capacity. (2) The end-customer demands at the retailer are observed and the holding or penalty costs are incurred at the retailer. (3) The retailer places an order with the supplier, if necessary, to reach the desired inventory level. (4) The supplier satisfies (the product will be available at the retailer at the start of the next period) the retailer demands to the best of her abilities. (5) If there is inventory left at the supplier, she incurs holding costs and on the other hand if there is some unsatisfied demand, it is supplied by expediting and the costs of expediting are incurred.

\subsection{The Traditional Model}

Here the retailer is using the $(s, S)$ policy that is optimal for her. The corresponding $s$ and $S$ values can be determined using an efficient solution procedure developed by Zheng and Federgruen (1991). In this setting the retailer does not order in every period, but informs the supplier about the end-customer demands. The non-stationary inventory control problem seen by the supplier was formulated and the relevant structural properties and solution procedures were described in detail in Gavirneni, kapuscinksi, and Tayur (1999).

\subsection{The Information Centric Model}

In this model we will consider a different operating policy at the retailer in the hope that the new operating policy will make better use of the information flow and thus improve the efficiency of the supply chain. Both the retailer and the supplier monitor the cumulative end-customer demand since the retailer last ordered. When this cumulative demand is greater than a predetermined value, $\delta$, then the retailer must place an order after the next end-customer demand. Thus, the supplier knows a period ahead when demand is going to occur, but is not sure of the size of the order. She has a probability distribution from which this demand will be realized.

After establishing the structure of the optimal policies, we developed simulation based procedure to compute the optimal parameters and costs (see Gavirneni 2002). In order to compute the impact of this information centric design on the supply chain performance, we performed a detailed computational study whose results are described below.

\subsection{Simulation Results}

The experimental setup for the study is as follows. The holding cost at the supplier is 1 while the penalty cost is allowed to take values 5,8 , and 11 . The retailer was also setup similarly. The end-customer demand is assumed to have a mean of 20 and was sampled from distributions Exponential(20), Erlang(2,10), Erlang(4,5), Erlang(8,2.5), and Erlang $(16,1.25)$. Thus the standard deviations of the end-customer demand were $20,14.2,10,7.1$, and 5 respectively. The production capacity at the supplier was allowed to take values 25,45 , and 65 . Thus the capacity was always greater than the mean demand. For all these cases we computed the costs of both the models. For each combination of parameters, we performed a search over some reasonable values of $\delta$. When the setup cost was greater than or equal to 10 , we used $\delta$ values ranging from 0 to 80 in multiples of 10 . When the setup cost was lower than 10 , we considered $\delta$ values from 0 to 10 in increments of 1 . Using a more exhaustive search can only result in an improved performance for the information centric model. The difference between the costs of these two models can be attributed to better usage of the information flows. For each case, we computed the percentage reduction as:

$$
100 \times \frac{\text { trad. model cost-info. centric model cost }}{\text { trad. model cost }}
$$


1. The Effect of Capacity The information centric model was more effective at higher values of supplier capacity. The main reason for this behavior is the flexibility that additional capacity provides the supplier. If the supplier is not able to (due to tight capacity) react to the more effective information flows, there would be no reduction in cost. Thus when the supplier has higher capacity, she is able to use the information flows efficiently and reduce her costs more significantly. Thus, the information centric strategy makes the supply chain more efficient at larger supplier capacities.

2. The Effect of Fixed Ordering Cost The average relative performance of the information-centric model as a function of the fixed ordering cost was tabulated. The fixed cost $K$ figures prominently in determining the optimal parameters for the two models. In the traditional model, the $s$ and $S$ are chosen in an optimal (at the retailer) fashion and for the information centric model, the fixed cost plays a role in determining the optimal $\delta$ value. We observed that, not surprisingly, at higher fixed costs, the optimal $\delta$ values were higher. We observed that the savings were lower at extreme values of fixed costs and it can be explained as follows. At higher fixed ordering cost, the retailer orders (less frequently) larger amounts, and the presence of finite capacity requires the supplier to start producing well ahead of time. This reduces her ability to react to unexpected changes at the retailer and the effectiveness of the information centric model is reduced. On the other hand, when the fixed costs are low, both models require that the retailer orders very frequently, thus reducing the difference in their performance. Thus, this strategy is most effective at moderate values of the fixed cost.

3. The Effect of Supplier and Retailer Penalty Costs Next we study how the savings of the information centric model are affected by the penalty costs at the supplier and the retailer respectively. We observed that the information centric model performs better at higher supplier penalty costs and at lower retailer penalty costs. We observed this behavior consistently among all the distributions. The main reason for this behavior is the way the costs at the retailer and the supplier change under the information centric model. Recall that under that model, the retailer is using a sub-optimal policy and her costs are increased while the costs at the supplier are decreased due to reduction in demand uncertainty. When her penalty costs are higher, the supplier realizes larger savings and the savings for the supply chain are higher. On the other hand, when the penalty costs at the retailer are higher, her costs under the new model increase more dramatically resulting in less effectiveness. Thus when the supplier penalty costs are high and the retailer penalty costs are low, the proposed information centric strategy is more effective.

4. The Effect of Demand Variance The average performance of the information centric model as a function of the standard deviation of the endcustomer demand is the focus here. We noticed that as the demand variance decreases the average performance of the proposed model increases. Recall that while the information centric model has no uncertainty about the timing of retailer demands, the quantity demanded is still uncertain. Thus when the end-customer demand has a high variance, the resulting uncertainty at the supplier is large even for the information centric model. Thus its performance is better at lower demand variance.

\section{A TWO-STAGE SERIAL SUPPLY CHAIN WITH A SIMPLE NEWSVENDOR AT EACH STAGE}

In the supply chain studied in this section, there is a single supplier with finite production capacity, $C$, supplying a single product to a newsvendor type retailer who is in turn facing independent and identically distributed demands (with cdf $\Psi(\cdot)$ and pdf $\psi(\cdot)$ ) from end-customers. The holding and penalty costs are respectively $h_{r}$ and $p_{r}$ at the retailer and $h_{s}$ and $p_{s}$ at the supplier. The costs and the demand distributions are known to both parties. There are no fixed ordering costs or lead times either at the retailer or the supplier. The unsatisfied demands at the retailer are backlogged and the unsatisfied demands at the supplier are sent to the retailer using an expediting strategy and $p_{s}$ represents the cost of expediting. Thus, if needed, the retailer can order and receive an infinite quantity of the product in a period. All these assumptions are common in inventory control literature and most of them, except the one on ordering costs, can be relaxed without significantly changing the general behavior of the system. Cachon and Zipkin (1999) studied a setting similar to this one. They used game theoretic models to study the impact on inventory levels of competition and cooperation between the retailer and the supplier.

We study this supply chain under a periodic setting and the sequence of events in every period is as follows: (1) The supplier decides (restricted by her capacity) how much to produce. The product is available immediately; (2) The retailer faces the end-customer demand and satisfies it to the best of her abilities. Unsatisfied demands are backlogged; (3) The retailer decides how much to order from the supplier; (4) The supplier satisfies the retailer's demand to the best of her abilities. Unsatisfied demands are supplied through the 


\section{Gavirneni}

expedited source. The product is available to the retailer at the beginning of the next period; (5) The holding and penalty costs at both the retailer and the supplier are computed and the problem goes to the next period. We measure the performance of this supply chain using the total holding and penalty costs at both the retailer and the supplier. Since the purchase costs between the retailer and the supplier are internal to the supply chain, they are not explicitly included in the total supply chain cost. The objective here is to study the effect of price fluctuations (at the supplier) and information sharing (between the retailer and the supplier) on the performance of this supply chain.

\subsection{Model Specifications}

We study the interaction between these two strategies in this supply chain by formulating and analyzing the retailer and supplier behavior in two different models. In the EDLP (every day low price) Model, the supplier charges the retailer the same price ( $c$ dollars per unit) in every period. In this setting, it is optimal for the retailer to use a stationary order up-to policy with the order up-to level $z$ in every period. Thus the end-customer demands at the retailer are transmitted to the supplier without any change and the supplier sees i.i.d. demands in every period. In every period, the supplier is completely aware of the inventory level at the retailer and there is no need for the retailer to provide additional information. In the HI-LO pricing Model, the supplier alternates the selling price between $c^{\prime}$ and $c^{\prime}-\epsilon$ from one period to the next. This leads to the retailer using an ordering pattern that repeats every two periods. In every cycle of two periods, the first period has an order up-to level $z^{\prime}$ while the second period has the order up-to level $z^{\prime}+\Delta_{\epsilon}$. Under this retailer inventory policy, the demands seen by the supplier are no longer i.i.d. We characterize the information (retailer inventory levels in each period) available to the supplier and formulate the resulting nonstationary inventory control problem she faces. Though the variance of demands seen by the supplier is increased, the benefits realized from the associated information flow will result in lower costs at her location. In addition, we will show that this reduction in costs at the supplier far outweighs the increase in the retailer's costs. Thus, if the supplier is willing to share some of the benefit she realizes with the retailer, the retailer may be willing to provide the inventory information and the whole supply chain will be more efficient.

As before, after establishing the structure of the optimal policies, we developed simulation based procedure to compute the optimal parameters and costs (see Gavirneni (2005)). In order to compute the impact of this information-centric design on the supply chain performance, we performed a detailed computational study whose results are described below. Percentage benefit was computed as in section 2 .

\subsection{Simulation Results}

The experimental setup for this study is as follows. The holding cost at the supplier is 1 while the penalty cost is allowed to take values 5, 8 , and 11 . The end-customer demand is assumed to have a mean of 20 and was sampled from distributions Exponential(20), Erlang(10,2), Erlang(5,4), and Erlang(2.5,8). Thus the standard deviations of the endcustomer demand were 20,14.1, 10, and 7.1 respectively. The production capacity at the supplier was allowed to take values 25,45 , and 65 . So, the capacity was always greater than the mean demand. For the EDLP model, the cost at the supplier was kept constant at five dollars per unit. In the HI-LO model, we let the cost at the supplier alternate between $5.0+K * 0.25$ and $5.0-K * 0.25$. We computed the total supply chain costs for values of $K$ ranging from 0 to 5 and chose the value of $K$ that resulted in the lowest total supply chain costs. Since the case $K=0$ represents the case of stationary policies, we know that this strategy can never result in increased supply chain costs. Computational results also demonstrate that, in many cases, the total costs of the supply chain were reduced. It is also possible to use a finer grid for the purchasing costs by changing the factor 0.25 to 0.1 . Since the key factor here is the difference in costs, the fact that we only consider symmetric fluctuations in selling prices does not significantly affect the results.

We report on the reduction in total supply chain costs when the supplier has information about the retailer inventory levels. In this setting, the total supply chain costs reduced by as much as $16.3 \%$ (average of 5.0\%). We now study how the supplier capacity, supplier penalty cost, and end-customer demand variance affect these benefits.

1. Effect of Supplier Capacity First we studied average percentage reduction as a function of supplier capacity and noticed that the benefits are consistently increasing as the capacity increases. This is because, when her capacity is not restrictive, the supplier is able to react to the information flows from the retailer. This enables her to realize higher benefits from these information flows, thus far eclipsing the inefficiencies (at the retailer) caused by the price fluctuations.

2. Effect of Supplier Penalty Cost The average percentage reduction is studied next as a function of the supplier penalty cost and the trend was clear in that the percentage benefit is higher at higher penalty costs. The reason for this is as follows: when there is tighter cooperation between the supplier and the retailer, the expediting necessary at the supplier is drastically reduced. Such a reduction has a higher benefit when the supplier penalty cost is high. 


\section{Gavirneni}

3. Effect of End-Customer Demand Variance The average percentage reduction as a function of the standard deviation of end-customer demand was analyzed next and it was evident that when the endcustomer demand is more variable, the percentage reduction is higher. This is due to the fact that when the demand has a variance, the information flows from the retailer are more beneficial. They are able to quickly alert the supplier of large swings in the end-customer demand.

\section{A DISTRIBUTION SUPPLY CHAIN WITH ONE SUPPLIER AND MANY RETAILERS}

In this section, we analyze a capacitated supplier, following a make-to-stock policy, providing a single product to $n$ retailers who are facing i.i.d. (in time) demands from the end-customers. The supplier has a finite production capacity, $C$, but has access to an alternate (possibly using overtime) expensive source that has infinite capacity. The difference in costs between these two modes of production is captured by her penalty cost $p_{s}$, and her holding cost is $h_{s}$. The retailers are all identical, face the same end-customer demand distribution, and have holding and penalty costs $h_{r}$ and $p_{r}$ respectively. There are no leadtimes, productions costs, or fixed ordering costs. The end-customer demand distribution, at each of the retailers, has cdf (pdf) $\Psi(\cdot)(\psi(\cdot))$ with mean $\mu$. Most of these assumptions are common in supply chain management and we believe they (except the one on fixed ordering costs) can be relaxed with little impact on the results.

\subsection{Model Specifications}

For this supply chain, we consider two models. In model 1, every retailer is allowed to order every period. Since there are no fixed costs between the retailers and the supplier, in every period, the supplier faces random demands from each of the retailers. If possible, she satisfies these demands from stock, and the unsatisfied demands are supplied (by incurring the penalty cost) from the alternate source. In model 2, the supplier specifies that retailer $j$ is allowed to order only in period $i n+j$ for $i \in\{0,1,2, \ldots\}$. In the periods that she is not allowed to order, she will receive a fixed quantity $\eta$. For this model, we assume that the retailers are sharing information (about their inventory levels) with the supplier. The supplier uses this information, especially from the retailers that are going to order in the immediate future, to determine the inventory level she wishes to maintain.

We studied both these models in detail, established the structure of optimal policies and developed simulation based solution procedures to compute the optimal parameters and costs. However, our focus here is the savings in supply chain costs and they were tabulated from an extensive com- putational study and the results are reported in the next section. Percentage difference in supply chain performance was computed as in section 2 .

\subsection{Simulation Results}

The experimental setup is as follows. The holding costs at the supplier and the retailers are set to 1 . The penalty costs at the retailers were equal to 9 while the penalty cost at the supplier was either 19,29, or 39. The end-customer demands have a mean equal to 20 , and were allowed to follow the distributions: Exponential(20), Erlang(2,10), Erlang(4,5), and Erlang $(8,2.5)$. Thus, demand variances were 400, 200, 100 , and 50 respectively. The number of retailers was either 2,3 , or 4 . The supplier capacity, when there were $n$ retailers, was set equal to $a \times n \times 20$ and $a$ was allowed to take values $1.5,2.0$, and 2.5. We also study two different conditions on the end-customer demands. In one case, the end-customer demands are assumed to be independent across the retailers. In another case, these demands are assumed to be highly $(\rho=1)$ positively correlated. One can easily guess that these scheduled ordering policies will be more beneficial when the demands at the retailers are positively correlated. All the same we decided to study the case of correlated demands to (1) observe how effective, in the best case, these strategies can be; and (2) get a better understanding of the effect of various system parameters on the effectiveness of these strategies. For each problem instance in model 2 , we determined the best possible $\eta$ value by performing an exhaustive search over values ranging from 0 to 40 in increments of 5. In all the cases the best $\eta$ value was either 20 or 25 , i.e. very close to the mean. Below, we detail our observations from this computational study. First, we study the cost per period and then the effectiveness of these strategies.

For the case of independent demands, this approach was effective in reducing the supply chain cost in 50\% of the problem instances. While in some cases the supply chain cost reduced by as much as $10.7 \%$, in other cases it increased by as much as $14.9 \%$. The average difference was an increase of $1.6 \%$. In the case of correlated demands, the supply chain cost recorded a decrease (ranging from $10.9 \%$ to $32.9 \%$ and averaging around $21.8 \%$ ) in all the cases. Thus we conclude that these strategies are beneficial in some cases of independent demands and their effectiveness grows when the demands are positively correlated.

1. Effect of Supplier Capacity First we study the percentage difference as a function of the supplier capacity parameter, $a$, for independent and correlated demands respectively. We noticed that in both the cases the percentage difference is increasing with increase in capacity. The principal reason for this behavior is that the supplier, when 
she has excess capacity, is more flexible to react to the information provided by the retailers. We conclude that these strategies are more useful when the supplier has excess capacity.

2. Effect of Supplier Penalty Cost The plots of the percentage difference as a function of the supplier penalty cost for independent and correlated demands were analyzed next. We observed that, in both the cases, as the supplier penalty cost increases percentage difference also increases. The reason for this behavior is that when the supplier penalty costs are high (relative to retailer penalty costs), the savings realized at the supplier using these strategies is greater than the resulting cost increases at the retailer. Thus we conclude that these strategies are more beneficial when the supplier penalty costs are high relative to those at the retailers.

3. Effect of End-customer Demand Variance The percentage difference as a function of the endcustomer demand variance for independent and correlated demands were also studied. The effect of variance was not immediately obvious. When the demands were independent, the percentage difference consistently decreased with decrease in the demand variance. On the other hand, in the presence of correlation between demands, the percentage difference appears to be highest at medium values of variance. When the demands are independent, the loss of benefits from risk pooling far outweigh the benefits from information sharing. Thus, there is a reduction in percentage difference as the demand variance decreases. When the demands are highly correlated, we know that loss of benefits from risk pooling is minimal and we must look at reduction in costs due to information sharing (which is lower at lower variances) and increase in costs due to infrequent retailer ordering (which is lower at extreme values of variance). Thus we conclude that these strategies are beneficial at higher variances when the demands are independent and at medium variances when the demands are correlated.

4. Effect of Number of Retailers The percentage difference for the cases of 2, 3, or 4 retailers was separately identified. We observed that, for independent demands, the percentage difference is highest when there are two retailers and it decreases as the number of retailers increases to 3 and then to 4. When the demands are positively correlated, we observed that the percentage difference is highest when there were three retailers. For the case when the demands are independent, the benefits lost from risk pooling are higher when the number of retailers is high. Thus in that case, these strategies are not as beneficial when there are more retailers. When the demands are correlated, there is not as much loss from the lack of risk pooling, but in the presence of a large number of retailers, the lowered frequency in the retailer ordering results in higher costs. Thus these strategies tend to lose their effectiveness when the number of retailers is high. So we conclude that these strategies are effective when the number of retailers is small (2 or 3 ).

\section{CONCLUSIONS \& FUTURE RESEARCH}

In this paper, via the use of three examples, we illustrated the benefits of information centric design and management of supply chains. Computational results from simulations of these supply chains have shown that supply chain performance can be significantly improved by the appropriate use of these strategies. While the supply chains studied here are representative of a wide array of supply chains, they by no means capture the complete spectrum. As a result, significant research activity is still needed in order to show that information centric strategies can be universally beneficial. We are currently involved in the following research projects intended to further expand the understanding behind managing information intensive supply chains:

1. In a multi-stage distribution supply chain, it is widely believed that well placed distribution centers can be effective in risk pooling, thus reducing uncertainties and improving supply chain performance. We wish to determine whether distribution centers continue to play an important role in supply chains in which information is readily available. We believe and wish to show that distribution centers do not carry such benefits in information-centric supply chains.

2. In a typical assembly supply chain, many suppliers ship parts or modules to a single location at which these parts or modules are assembled into the final product. Traditionally, in such supply chains, a supplier has a localized perspective and is not aware of the status of the other suppliers. This often leads to a lack of coordination and results in major inefficiencies in the system. It is possible for the various suppliers to share information, so that the decisions are made in a coordinated manner. However, it is not easy to determine which information should be provided to whom and how this information should be used. We wish to explore these possibilities and come up with strategies for managing these supply chain efficiently. 


\section{REFERENCES}

Armistead, C.G. and Mapes, J., 1993. "The impact of supply chain integration on operating performance," Logistics Information Management, 6(4): 9-14.

Cachon, G. and P. Zipkin, 1999. "Competitive and cooperative inventory policies in a two stage supply chain," Management Science, 45(7): 936-953.

Gavirneni, S., Kapuscinski, R. and S. Tayur, 1999. "Value of information in capacitated supply chains," Management Science, 45(1): 16-24.

Gavirneni, S., 2002. "Information flows in capacitated supply chains with fixed costs," Management Science, 48(5): 644-651

Gavirneni, S., 2005. "Price fluctuations, information sharing, and supply chain performance," Working Paper, Cornell University.

Gavirneni, S., and S. Tayur, 1999. "Managing a single customer using a target reverting policy," Manufacturing \& Service Operations Management Journal, 1(2): 157173.

Lee. H., Padmanabhan. P., and S. Whang, 1996. "Information distortion in a supply Chain : The bullwhip effect," Management Science, 43(4): 546-558.

Lewis, B., 2001. "The seventy percent failure rate," Info World, 23(44): 50-50.

Takac, P.F., 1992. "Electronic data interchange (EDI): an avenue to better performance and the improvement of trading relationships?," International Journal of Computer Applications in Technology, 5(1): 22-36.

Zheng, Y.S. and A. Federgruen, 1991. "Finding optimal $(s, S)$ policies is about as simple as evaluating a single policy," Operations Research, 39(4): 654-665.

\section{AUTHOR BIOGRAPHY}

SRINAGESH GAVIRNENI is an assistant professor of operations management in the Johnson Graduate School of Management at Cornell University. His research interests are in the areas of supply chain management, inventory control, production scheduling, simulation, and optimization. His papers have appeared in Management Science, Operations Research, Manufacturing \& Service Operations Management, European Journal of Operational Research, Operations Research Letters, IIE Transactions, Interfaces, and IEEE Transactions on Reliability. Previously, he was an assistant professor in the Kelley School of Business at Indiana University. Before that he was the chief algorithm design engineer of SmartOps, a Software Architect at Maxager Technology, Inc., and a research scientist with Schlumberger. His undergraduate degree from IIT-Madras is in Mechanical Engineering and he has received Masters degrees from Iowa State University and Carnegie Mellon University. His email address is <sg337@cornell. edu $>$. 